\title{
SOFT COMPUTING BASED MEDICAL IMAGE RETRIEVAL USING SHAPE AND TEXTURE FEATURES
}

\author{
${ }^{1}$ M. Mary Helta Daisy and ${ }^{2}$ S. Tamil Selvi \\ ${ }^{1}$ Department of E.C.E, St. Xavier's Catholic College of Engineering, Chunkankadai, India \\ ${ }^{2}$ Department of E.C.E, National Engineering College, Kovilpatti, India
}

Received 2013-10-06; Revised 2013-11-03; Accepted 2013-12-24

\begin{abstract}
Image retrieval is a challenging and important research applications like digital libraries and medical image databases. Content-based image retrieval is useful in retrieving images from database based on the feature vector generated with the help of the image features. In this study, we present image retrieval based on the genetic algorithm. The shape feature and morphological based texture features are extracted images in the database and query image. Then generating chromosome based on the distance value obtained by the difference feature vector of images in the data base and the query image. In the selected chromosome the genetic operators like cross over and mutation are applied. After that the best chromosome selected and displays the most similar images to the query image. The retrieval performance of the method shows better retrieval result.
\end{abstract}

Keywords: Medical Image, Genetic Algorithm, Image Retrieval

\section{INTRODUCTION}

Content based image retrieval is applied to visual contents for search images from the feature databases. In the medical field enormous images are produced and used for diagnostic purposes (Daisy and Selvi, 2012). The visual and multimedia data are steadily increasing. So, there is a need for fast retrieval methods apart from textbased retrieval. To search large multimedia databases for retrieval purposes, the visual and audio content use commonly (Muller et al., 2004). The visual content of images like color, texture and shape are use by the CBIR system. The unified approach usually have extraction of visual features, feature extraction, classification and similarity measure (Jaganathan and Vennila, 2013).

The genetic algorithm is introduced to do the optimization, genetic algorithm to evolve chromosome population using the various genetic operations like selection, crossover and mutation. The objective of the selection and reproduction operator is to keep copies of best element in the chromosome to propagate for next generation. The fitness function is used to evaluate the chromosome. The crossover operator creates a new structure by exchanging element in the chromosome (Cuevasa et al., 2002). Optimization of CBIR is a time consuming task because the entire image in the database indexed again when each time the indexing algorithm is use for that purpose (Saadatmand-Tarzjan and Moghaddam, 2007). The crossover operator exchanges the subset of genes with pair of individuals and generates two others. Mutation operator replaces randomly selected genes from an individual (Santos et al., 2008).

The genetic algorithm application begins with initial population and the individuals which are randomly generated. The fitness value of the each chromosome is evaluated and determines the appropriateness of the problem. The individual selected from the population before the recombination are called parent. After the recombination the chromosome are called as the children (Cho and Lee, 2002). The genetic cross over is applied to the feature vectors. The new chromosome after the crossover and feature vector in the database are compared based on similarity measure. The most similar chromosome are use for the next generation (Yoo and Cho, 2007). Corresponding Author: M. Mary Helta Daisy, Department of E.C.E, St. Xavier's Catholic College of Engineering, Chunkankadai, India 
The remaining part of the paper is structured as follows, section 2 presents the related work, section 3 concept concerning the work, section 4 explain the proposed work, section 5 presents result and discussion, section 6 address the conclusion of this research.

\section{MATERIALS AND METHODS}

Saadatmand-Tarzjan and Abrishami (2007) was presented optimization of CBIR using Evolutionary Group Algorithm (EGA). In this approach the image database subdivided in to small groups. GA use the small group for evaluation. The chromosome consists of genes. The chromosome has evolutionary genes and history genes. EGA shows good precision and recall values.

Santos et al. (2008) proposed GA based image retrieval. This method considering the local features of the image. By considering the combination of the right features of the image together to get suitable results. The user preference and similarity uses GA.

Cho and Lee (2002) proposed Interactive GA for retrieval system. Wavelet transform are used for the feature extraction from the images. This method provides the user friendliness to retrieve images from the large databases. This enable the implicit queries rather than the explicit queries.

Gali et al. (2012) have proposed GA for content based image retrieval system. The low level image features like color, texture and shape are combined and trained with different weight values. To evaluate and optimize features weights of CBIR coded chromosome used. The optimum weight of the feature calculated with better fitness function.

Lai and Chen (2009) have proposed interactive genetic algorithm for CBIR. They combined the color feature and texture feature for the retrieval purposes. The differences of the retrieved result and user query are adjusted by the weight of color and texture features based on the expectation of the user.

Raghuwanshi et al. (2012) have presented implicit and explicit feedback based image retrieval using genetic algorithm. The combining approach improves the user perception of the image resemblance. It provides two tier architecture. The color and texture features are considered as low level features for image retrieval.

Torres et al. (2009) proposed GA framework for the combination of the similarity measure was presented. The GA is more suitable for combining the simple descriptors of CBIR, which similar images returned from the database.

Ferreira et al. (2008) presented GA based image retrieval using the relevance feedback. This approach consider the color, texture and shape feature of an image in the data base for the retrieval purpose. The GA find out the better combination of the feature descriptor to produce similar images.

Syam and Rao (2012) proposed GA for CBIR using extensive feature. The extensive feature vectors of the contour, texture and color of the image are extracted. The GA based approach is applied both query and database images. Then the retrieval accuracy is evaluated by the precision and recall measure.

Hatwar and Hatwar (2012) proposes GA based retrieval for the relevance feedback. The query image and database images are segmented first. The texture and color feature of the images are extracted to form the feature vector for the comparison. By considering the three regions of the image are selected and combining the three to form the chromosome of the genetic algorithm.

\subsection{Feature Extraction}

As per the proposed technique, 2 features can be computed from each region of an individual image in a database. The calculated features are stored individually in a database. The two features are (Daisy and Selvi, 2012):

- Texture feature

- Shape feature

\subsection{Texture Feature}

The texture feature provides the better image description of the visual content. The texture analysis can make use of the different attributes like segmentation and recognition. For the feature extraction purpose different approaches like statistical and structural are considered by CBIR. The structural method describes the texture as the primitive form. The texture features usually provides the smoothness and coarseness of the image. So many types of method describe the texture as the primitive form. The texture features usually provides the smoothness and coarseness of the image. So many types of methods for computing the texture feature of the image. But no one is suitable to all the varieties of the features.

\subsection{Gabor Filters}

Gabor filter can be represented by the following equation in the spatial domain as Equation (1).

The Equation (1) is the representation of the Gabor filter:

$\mathrm{G}_{\sigma, \varphi, \theta}(\mathrm{x}, \mathrm{y})=\mathrm{g}_{\sigma}(\mathrm{x}, \mathrm{y}) \cdot \exp [2 \pi \mathrm{j} \varphi(\mathrm{x} \cos \theta+\mathrm{y} \sin \theta)]$ 
Where:

$\mathrm{g}_{\sigma}=\frac{1}{2 \pi \sigma^{2}} \exp \left[\left(\mathrm{x}^{2}+\mathrm{y}^{2}\right) / 2 \sigma^{2}\right]$

The Gabor filter $G_{\sigma, \varphi, \theta}(x, y)$ forms the complex valued function. Decomposing $\mathrm{G}_{\sigma, \varphi, \theta}(\mathrm{x}, \mathrm{y})$ leads into real and imaginary parts in Equation (2):

$$
\mathrm{G}_{\sigma, \varphi, \theta}(\mathrm{x}, \mathrm{y})=\mathrm{R}_{\sigma, \varphi, \theta}(\mathrm{x}, \mathrm{y})+\mathrm{jI}_{\sigma, \varphi, \theta}(\mathrm{x}, \mathrm{y})
$$

Where:

$$
\begin{aligned}
& R_{\sigma, \varphi, \theta}(x, y)=g_{\sigma}(x, y) \cos [2 \pi \varphi(x \cos \theta+y \sin \theta)] \\
& I_{\sigma, \varphi, \theta}(x, y)=g_{\sigma}(x, y) \sin [2 \pi \varphi(x \cos \theta+y \sin \theta)]
\end{aligned}
$$

\subsection{Shape Feature}

The shape features of the image can be the gray scale equivalent of every image in the database. Human can perceive the scenery which composed of many objects together. The object can use for the better identification of their shapes. The shape is one of the features for the user to describe as query.

\subsection{Fourier Descriptor}

Fourier transformation mainly used for shape analysis. It can able to represent the object in the frequency domain. The common general features of the shape can be represented by lower frequency of the Fourier transformation. The important details of the shape are representing by the higher frequency of the Fourier descriptor. Fourier transform usually generate large number of coefficients. The discrete Fourier transform of $\mathrm{s}(\mathrm{t})$ is given by Equation (3):

$\mathrm{u}_{\mathrm{n}}=\frac{1}{\mathrm{~N}} \sum_{\mathrm{t}=0}^{\mathrm{N}-1} \mathrm{~s}(\mathrm{t}) \exp \left(\frac{-\mathrm{j} 2 \pi \mathrm{nt}}{\mathrm{N}}\right), \mathrm{n}=0,1, \ldots, \mathrm{N}-1$

Here $\mathrm{u}_{\mathrm{n}}, \mathrm{n}=0,1, \ldots, \mathrm{N}-1$, are usually called coefficients of the shape of Fourier descriptors, denoted as $\mathrm{FD}_{\mathrm{n}}, \mathrm{n}=0,1, . ., \mathrm{N}-1$.

\subsection{Algorithms}

Algorithm I (Generation of feature vector from database image and query image):

1. Convert the input image to gray scale
2. Apply Gaussian filter to blur the images in database and query

3. Apply Fourier descriptor operation to extract the shape feature

4. Apply Gabor filter operation to the i-th image for extracting texture feature

5. Apply morphological operator for opening texture feature for further operation

6. Concatenate the shape and texture feature values to get the feature vector of $i$-th image

7. Repeat the step 2 to step 6 for the query image and extract query image feature vector (Daisy and Selvi, 2012):

- Gene: It is the difference of the feature vector value of the image in the database and query image

- Chromosome: Each chromosome consists of 10 genes

- Distance value: This is the sum of the gene in a given chromosome

- Cross over: Crossover is one of the genetic operators. It mates two chromosomes to generate a new offspring

- Mutation: Mutation is also one of the genetic operators. That make change one or more gene value in the chromosome from its starting state

Algorithm II (Genetic based image retrieval):

1. Generate 10 chromosomes without repeating the same gene once again in it

2. Find the distance value of all chromosomes

3. Select the best five chromosomes (n1) based on the distance value to perform crossover and mutation

4. After genetic operation get the five chromosomes (n2)

5. Combine the $\mathrm{n} 1$ and $\mathrm{n} 2$ chromosome to use next generation

6. Repeat the steps 2 to 5 for ten generations

7. At the end of the ten generation select the best chromosome based on the minimum distance value

8. Display the images based on the genes in the selected chromosome

\section{RESULTS}

The proposed approach contains a database of 1500 images. This includes 500 brain images, 500 liver images and 500 lung images. The experiments were conducted using MATLAB. The efficiency of the retrieval accuracy is measured by precision and recall measure. By 
considering the genetic algorithm and without genetic algorithm, the precision and recall is estimated. The precision and recall of the method based genetic algorithm outperforms the method considering without genetic algorithm. The precision and recall mentioned in the proposed method which are computed for a given query images and the associated precision-recall graph is shown in Fig. 3-5. The retrieved images and the precision-recall graph show that the proposed CBIR system claims effectiveness in retrieving images that are most similar to the given query image.

The retrieval performance of the soft computing based method is analyzed by means of the precision and recall. The precision and recall are defined as follows:

$$
\begin{gathered}
\text { precision }=\frac{\text { Number of retrieved images relevant to the query image }}{\text { Total number of images retrieved }} \\
\text { recall }=\frac{\text { Number of retrieved images relevant to the query image }}{\text { Total number of relevant images in the database }}
\end{gathered}
$$

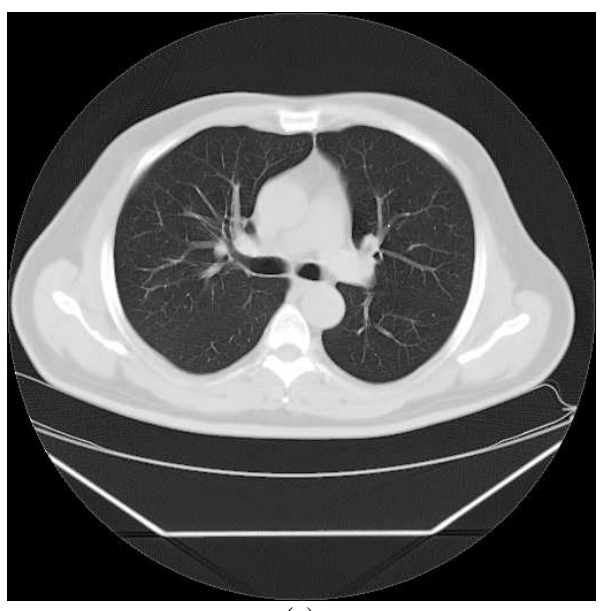

(a)
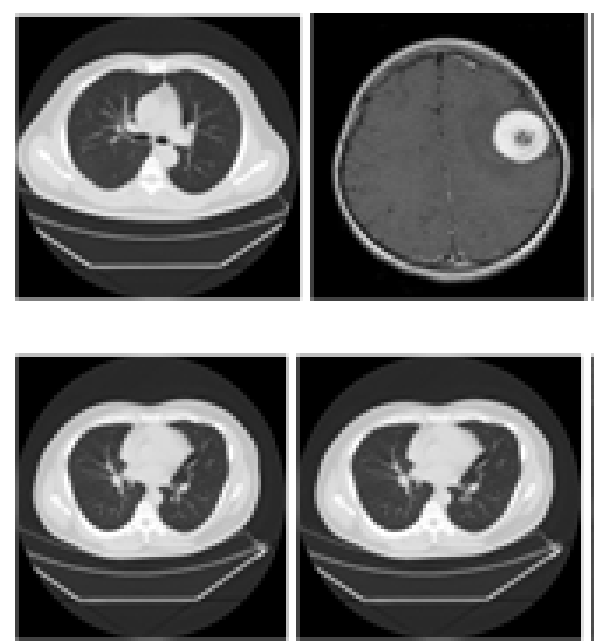

(c)
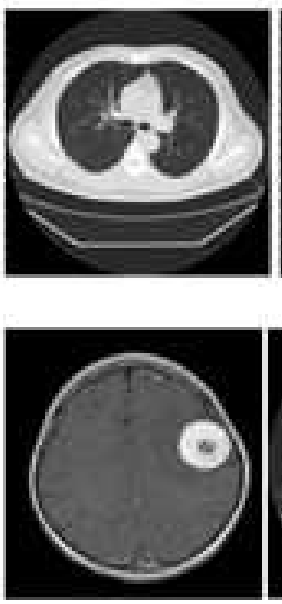
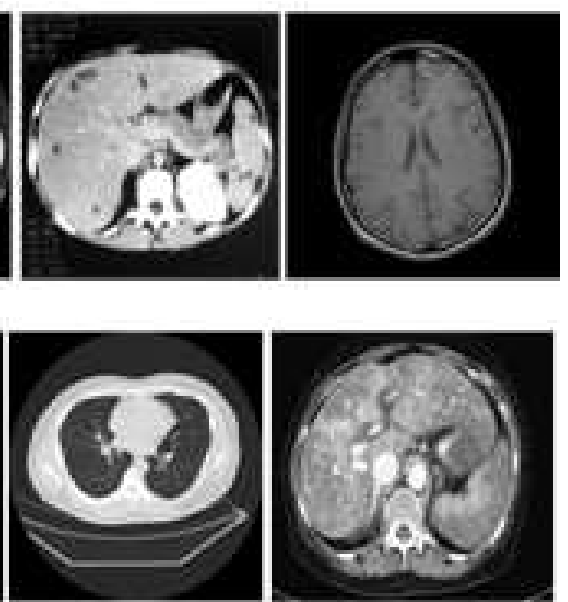

(b)
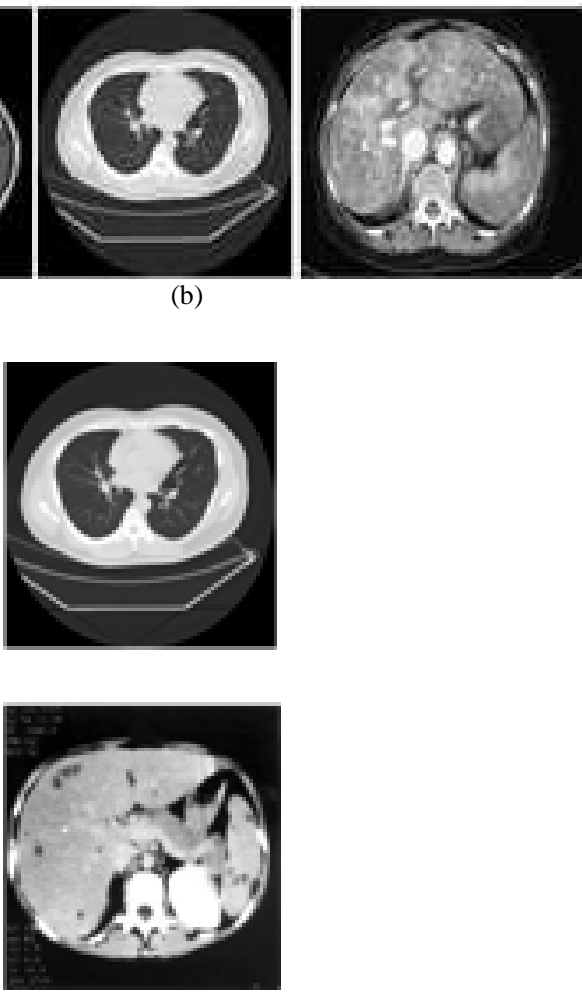

Fig. 1. Sample output obtained for Lung image (a) query image (b) retrieved images without GA (c) retrieved images with GA 
M. Mary Helta Daisy and S. Tamil Selvi / American Journal of Applied Sciences 11 (2): 258-265, 2014

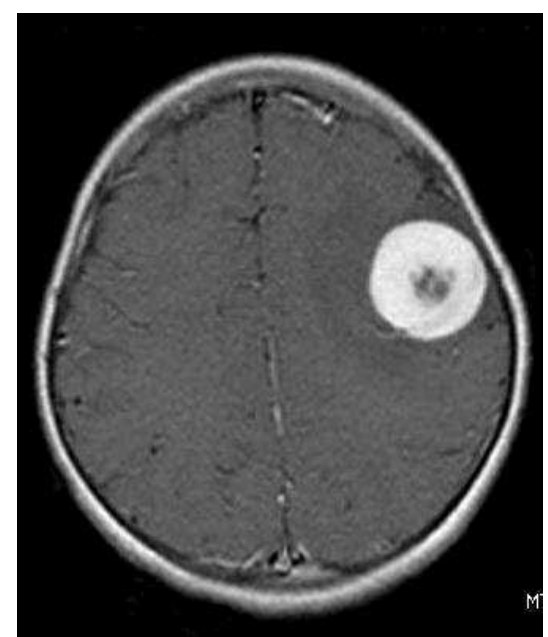

(a)
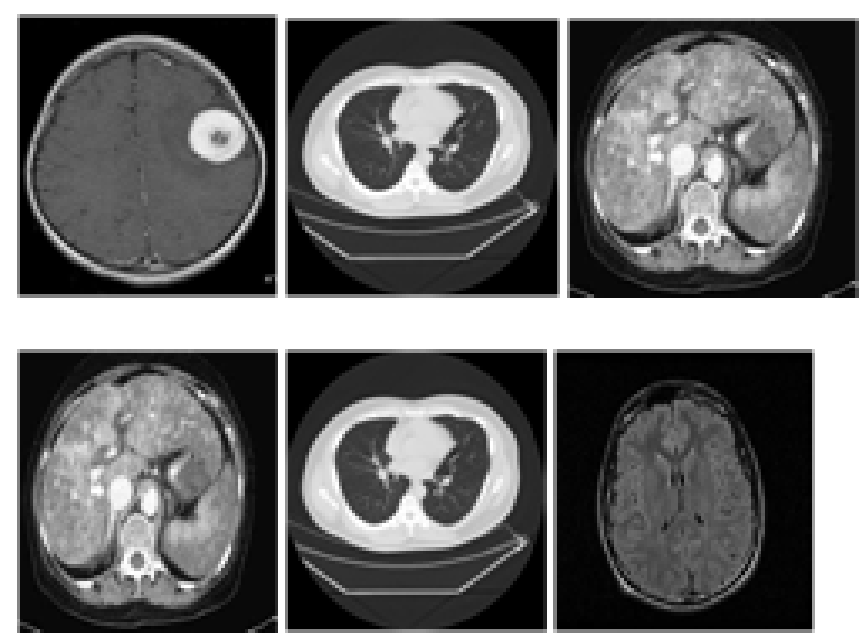

(b)
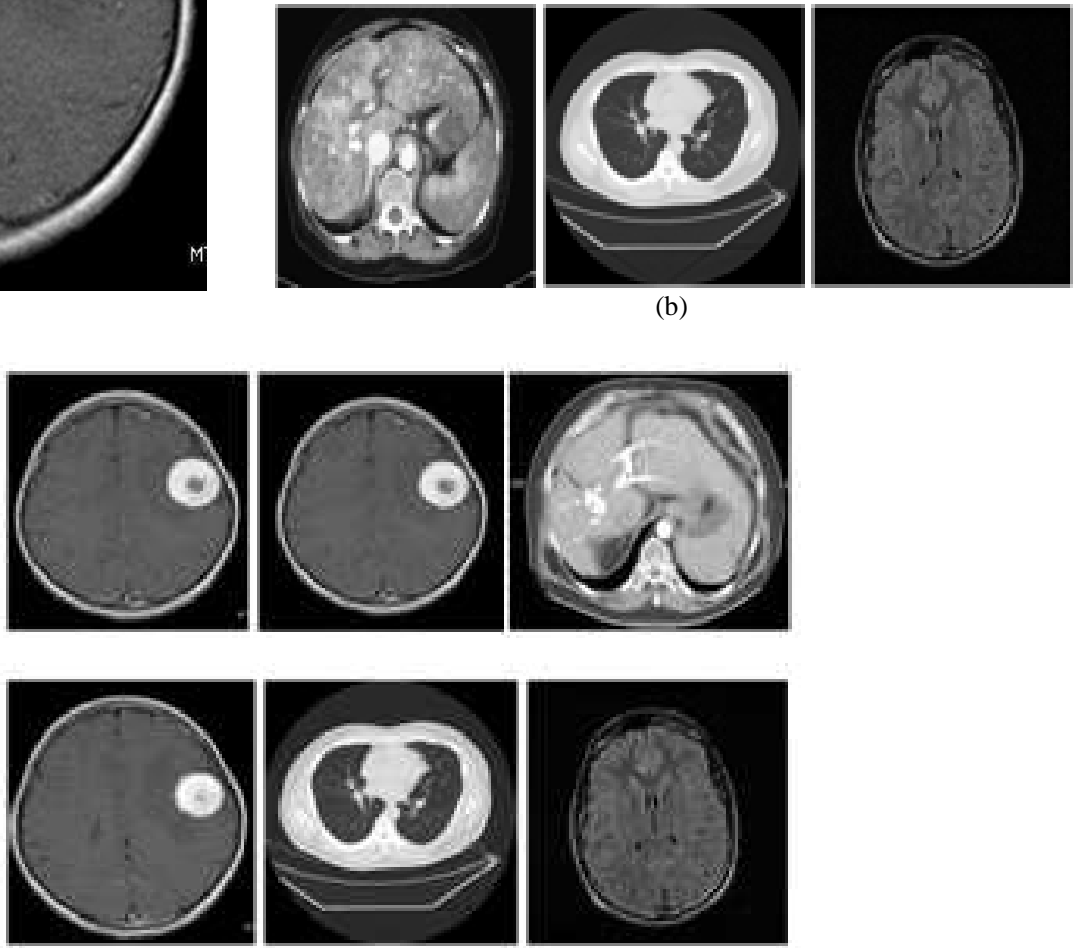

(c)

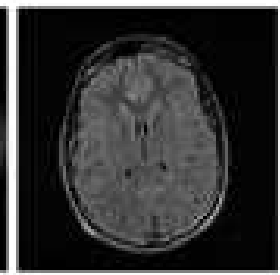

Fig. 2. Sample output obtained for Brian MRI image (a) query image (b) retrieved images without GA (c) retrieved images with GA

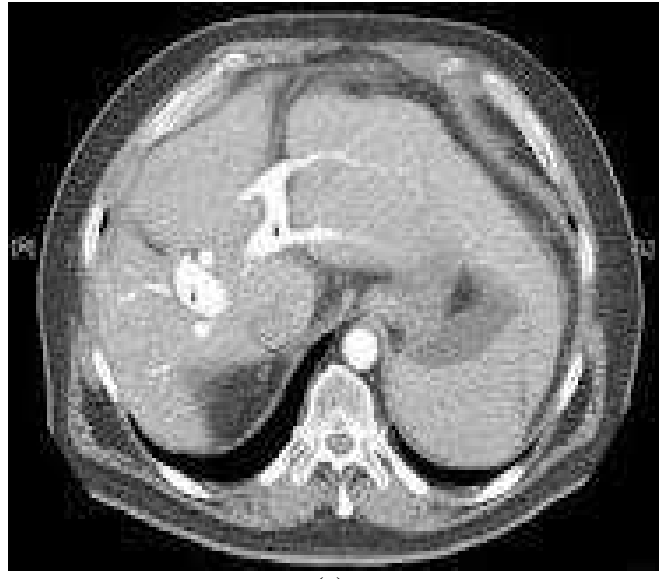

(a)
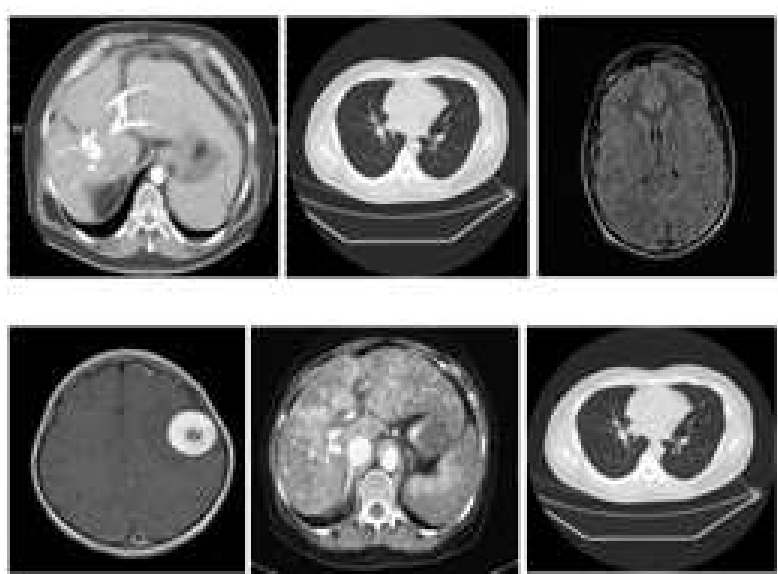

262

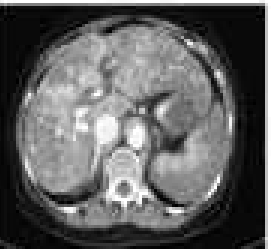

(b)

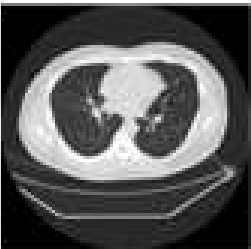



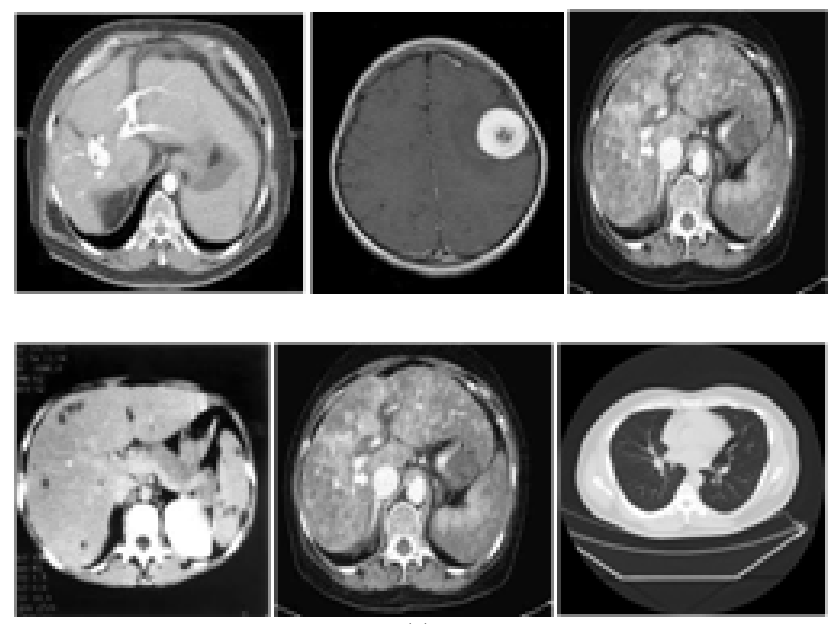

(c)

Fig. 3. Sample output obtained for Liver image (a) query image (b) retrieved images without GA (c) retrieved images with GA

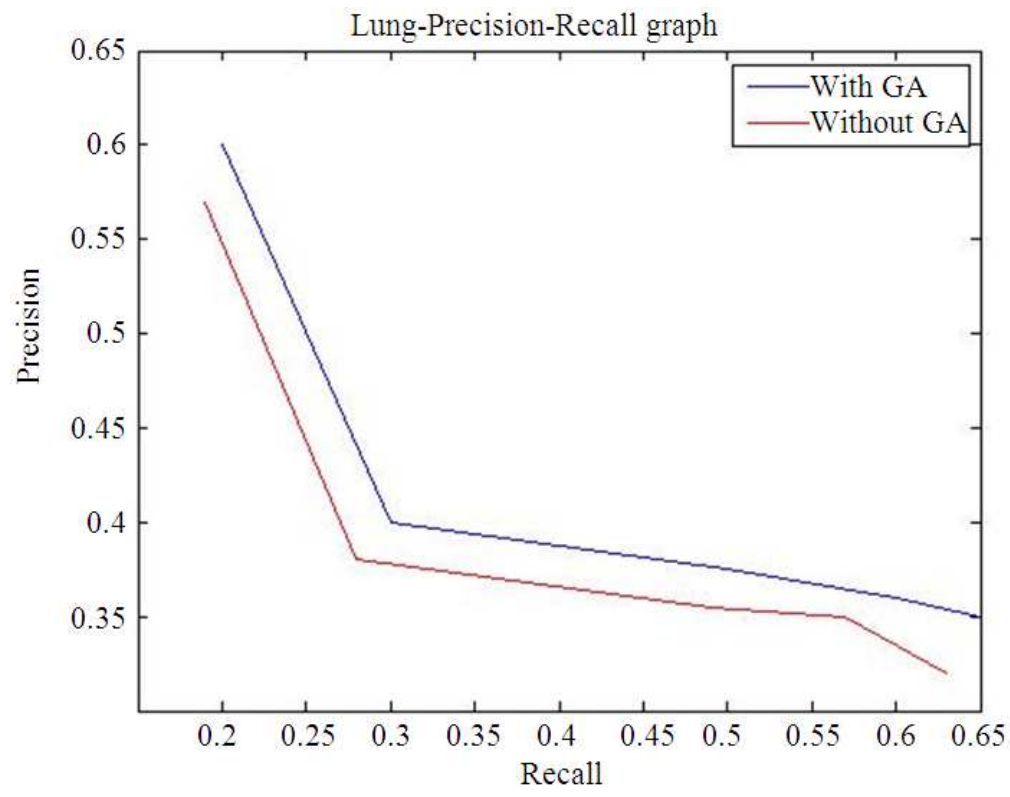

Fig. 4. Performance comparison for lung images

The Fig. 1 shows the query image and the output image of the lung. Figure $\mathbf{1 b}$ is the retrieved images using without soft computing technique. This collection consists of more number of non lungs images. Figure 1c is the collection of retrieved images based on the soft computing method. This consists of more images related to the query image. The method based on genetic algorithm has provided better retrieval accuracy. The comparison graph Fig. 4 shows that the soft computing based method gives better precision and recall ratio.
Figure 2 represents the query and retrieved images of the brain. While comparing both collection shown in Fig. $\mathbf{2 b}$ and 2c. The images retrieved based on genetic algorithm has contain more query related images. The graph Fig. 5 shows the proposed method perform better than the method not using soft computing.

Figure 3 shows the query and retrieved output images of the liver. The comparison graph Fig. 6 shows the genetic algorithm based method provide better precision and recall ratio. 


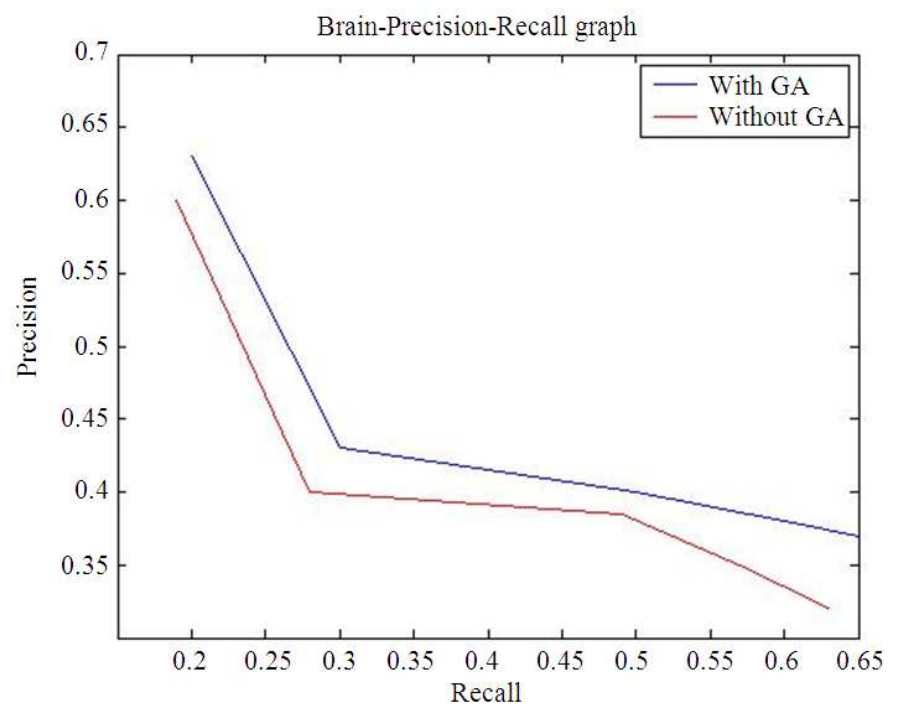

Fig. 5. Performance comparison for brian images

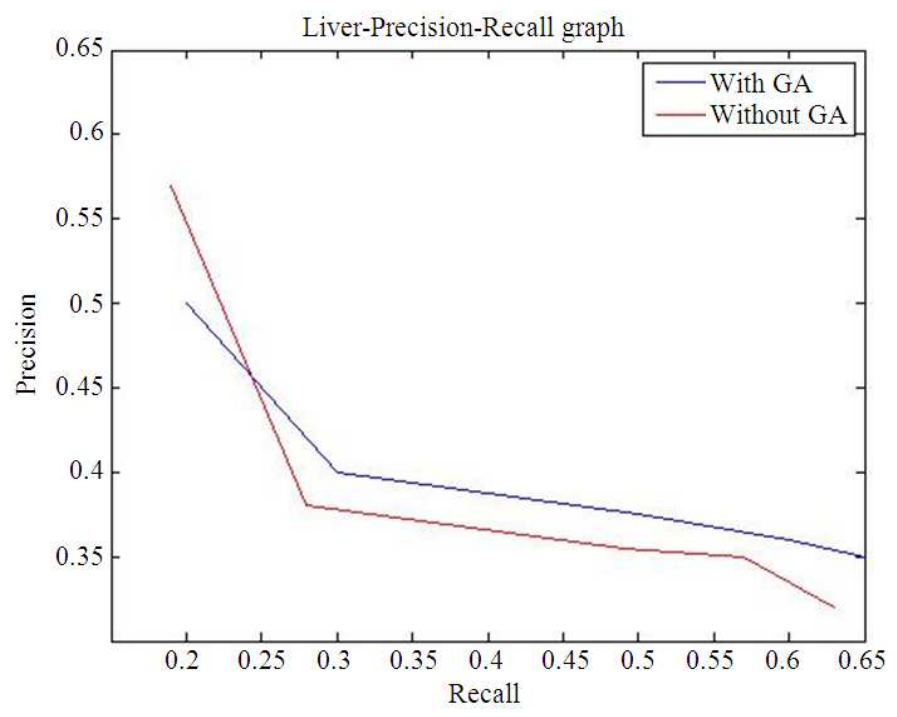

Fig. 6. Performance comparison for liver images

\section{DISCUSSION}

The precision and recall measures are evaluate the performance of the proposed method with traditional one. Vilvanathan and Rangaswamy (2013) considered with classification method using content based image retrieval with color feature extraction. The optimization method like genetic algorithm proposed in our work with respect to the medical images. The content based medical image retrieval framework proposed in (Maheswari,
2013), the brain image only considered. In the proposed work three different medical images like lungs, brain and liver are considered and the genetic based soft computing method based retrieval used.

\section{CONCLUSION}

We proposed CBIR based medical image retrieval using genetic algorithm for retrieving relevant images from image database. This approach extracted the 
features like shape and morphological based texture from the image using Fourier descriptor, Gabor filter respectively. The cross over and mutation operators are applied to the chromosomes. The best chromosome is selected then displays the related images from the database with respect to query image. The retrieved images gave better result. In this study we considered 1500 medical images, considered the texture and shape for the feature extraction purpose. In future works the data base images can be increased to sufficient numbers, the various features combinations use for the feature extraction for getting more accurate precision, recall ratio.

\section{REFERENCES}

Cho, S.B. and J.Y. Lee, 2002. A human-oriented image retrieval system using interactive genetic algorithm. IEEE Trans. Syst. Man Cybernet., 32: 452-458. DOI: 10.1109/TSMCA.2002.802812

Cuevasa, F.J., J.H. Sossa-Azuelab and M. Servin, 2002. A parametric method applied to phase recovery from a fringe pattern based on a genetic algorithm. Opt. Commun., 203: 213-223. DOI: 10.1016/S00304018(02)01132-X

Daisy, M.M.H. and S.T. Selvi, 2012. Content based MRI brain image retrieval by combining Fourier descriptors and Gabor filters with morphological operation. Eur. J. Scient. Res., 87: 469-478.

Ferreira, C.D., R.D.S. Torres, M.A. Goncalves and W. Fan, 2008. Image retrieval with relevance feedback based on genetic programming. Proceedings of the 23rd Brazilian symposium on Databases, Oct. 13-15, ACM Press, Porto Alegre, Brazil, pp: 120-134.

Gali, R., M.L. Dewal and R.S. Anand, 2012. Genetic algorithm for content based image retrieval. Proceedings of the 4th International Conference on Computational Intelligence, Communication Systems and Networks, Jul. 24-26, IEEE Xplore Press, Phuket, pp: 243-247. DOI: 10.1109/CICSyN.2012.52

Hatwar, N.R. and R.F. Hatwar, 2012. Content based image retrieval genetic algorithm for relevance feedback. Int. J. Adv. Comput. Res., 2: 381-385.

Jaganathan, Y. and I. Vennila, 2013. Feature dimension reduction for efficient medical image retrieval system using unified framework. J. Comput. Sci., 9: 1472-1486. DOI: $10.3844 /$ jcssp.2013.1472.1486

Lai, C.C. and Y.C. Chen, 2009. Color Image Retrieval Based on Interactive Genetic Algorithm. In: NextGeneration Applied Intelligence, Chien, B.C., T.P. Hong, S.M. Chen and M. Ali (Eds.), Springer, Berlin, ISBN-10: 3642025676, pp: 343-349.
Maheswari, U., 2013. Content based medical image retrieval using binary association rules. J. Comput. Sci., 9: 678-689. DOI: 10.3844/jcssp.2013.678.689

Muller, H., N. Michoux, D. Bandon and A. Geissbuhler, 2004. A review of content-based image retrieval systems in medical applications-clinical benefits and future directions. Int. J. Med. Inform., 73: 1-23. PMID: 15036075

Raghuwanshi, G., N. Mishra and S. Sharma, 2012. Content based image retrieval using implicit and explicit feedback with interactive genetic algorithm. Int. J. Comput. Applic., 43: 8-14.

Saadatmand-Tarzjan, M. and H.A. Moghaddam, 2007. A novel evolutionary approach for optimizing contentbased image indexing algorithms. IEEE Trans. Syst. Man Cybernet., 37: 139-153. DOI: 10.1109/TSMCB.2006.880137

Santos, J., C.D. Ferreira and R. Torres, 2008. A genetic programming approach for relevance feedback in region-based image retrieval systems. Proceedings of the 21th Brazilian Symposium on Computer Graphics and Image Processing, Oct. 12-15, IEEE Xplore Press, Campo Grande, pp: 155-162. DOI: 10.1109/SIBGRAPI.2008.15

Syam, B. and Y. Rao, 2012. An effective similarity measure via genetic algorithm for content-based image retrieval with extensive features. Int. J. Signal Imag. Syst. Eng., 5: 18-28. DOI: 10.1504/IJSISE.2012.046742

Torres, R.DS., A.X. Falcao, M.A. Goncalves, J.P. Papa and B. Zhang et al., 2009. A genetic programming framework for content-based image retrieval. Patt. Recogn., 42: 283-292. DOI: 10.1016/j.patcog.2008.04.010

Vilvanathan, K. and R. Rangaswamy, 2013. Bi-level classification of color indexed image histograms for content based image retrieval. J. Comput. Sci., 9: 343-349. DOI: 10.3844/jessp.2013.343.349

Yoo, H.W. and S.B. Cho, 2007. Video scene retrieval with interactive genetic algorithm. Multimedia Tools Applic., 34: 317-336. DOI: 10.1007/s11042007-0109-8 